\title{
Prevalence and prognostic factors for erectile dysfunction in renal transplant recipients
}

\author{
Jaime A. Wong, MD; ${ }^{*}$ Joseph Lawen, MD; ${ }^{*}$ Bryce Kiberd, $M D ;{ }^{\dagger}$ Waleed K. Alkhudair, MD*
}

\begin{abstract}
Introduction: The purpose of this study was to determine the prevalence of erectile dysfunction (ED) at our institution in the postrenal transplant population and to compare those patients who had ED with those who did not have ED, with respect to several patient characteristics.

Methods: We conducted a cross-sectional study of male renal transplant recipients who were in attendance at the transplant clinic from April 1, 2004, to March 31, 2006. Erectile function was evaluated using the International Index of Erectile Function short form questionnaire. Patients were also screened for depression using the Beck Depression Inventory. We performed a chart review to obtain various patient characteristics.

Results: This study involved 55 patients. Their average age was 50 years old and the mean duration of the current transplant was 7.9 years. ED was identified in 28 of the patients (51\%). More patients with ED were over age 50 years $(64 \%$ v. $26 \%, p=0.004)$. There was a higher prevalence of diabetes mellitus (39\% v. $11 \%, p=0.02)$ in patients with ED compared with those patients without ED. More patients with ED were depressed compared with those patients who did not have ED ( $29 \%$ v. $7 \%, p=0.04)$. These 3 factors were significantly associated with ED and this relationship was confirmed on multivariate analysis.
\end{abstract}

Conclusion: ED remains a common problem in the renal transplant population. The cause of ED is multifactorial, with increasing age and the presence of diabetes mellitus and depression increasing the risk of ED.

CUAJ 2007;1(4):383-7

\section{Introduction}

Renal failure is a risk factor for erectile dysfunction (ED). ${ }^{1}$ The cause of $\mathrm{ED}$ in patients with renal failure is usually multifactorial, with potential contributing factors including those that are vasculogenic, neurologic, endocrinologic, psychological, biochemical and pharmacologic. ${ }^{2}$ Neuroendocrine disturbances involving the hypothalamic-pituitarygonadal axis are not reversed with dialysis but are improved by renal transplantation. ${ }^{1}$ Psychogenic factors may also have an effect on erectile function in patients with renal failure as about one-quarter of these patients are depressed at any given time. ${ }^{3}$ Changes in lifestyle, including dietary restriction, polypharmacy and hemodialysis, predispose dialysis patients to depression. ${ }^{3}$
$\mathrm{ED}$ is a common disorder in men aged 40 years and over. Prevalence of ED increases with age, with $25 \%, 55 \%$ and $65 \%$ of men aged 65,75 and 80 years old, respectively, reporting the disorder. ${ }^{4}$ In a cross-sectional study of sexual function of male kidney transplant recipients, ED was considerably more common than it was in the control population, with a prevalence of $55.7 \%$ despite the transplant recipients being younger than those in the control group. ${ }^{5}$ In that study, age, time on dialysis, and iterative transplants were significantly and negatively related to erectile dysfunction.${ }^{5}$ The increased number of kidney transplants being performed in addition to the improved graft and patient survival rates has resulted in a greater number of patients with ED. ${ }^{4}$ Thus, there is an increasing number of men experiencing a major quality of life issue. ${ }^{4}$

The literature regarding the effect of renal transplantation on patients with ED has been contradictory. In most studies, patients regain potency following renal transplant. ${ }^{2}$ However, in one longitudinal study, ED did not improve markedly after transplantation. ${ }^{6}$ In other cases, particularly in patients who received second or subsequent transplantations, erectile function has been adversely affected by interference with penile arterial blood flow. ${ }^{6}$

The purpose of this study was to determine the prevalence of $\mathrm{ED}$ at our institution in the postrenal transplant population. In addition, we compared several patient characteristics of patients in this population who had ED with those postrenal transplant patients who did not have ED.

\section{Methods}

We conducted a cross-sectional study of erectile function in male renal transplant recipi- 
ents. All male posttransplant patients aged 18 years or older who were at least 1 year post renal transplant and were in attendance at the transplant clinic at our institution from April 1, 2004, to March 31, 2006, were eligible for this study. Erectile function was evaluated using the International Index of Erectile Function short form questionnaire (IIEF-5). ${ }^{7}$ This is a brief, self-administered questionnaire that consists of 5 questions and was designed to address 5 relevant aspects of erectile function .? The IIEF- 5 score ranges from 5 to 25 . A cutoff score of 21 best discriminates the presence of ED, with a sensitivity of 0.98 and a specificity of $0.88 .^{7}$ This questionnaire has been validated in the postrenal transplant population. ${ }^{5,7}$ With the IIEF-5, ED is classified into 5 severity levels: none (22-25), mild (17-21), mild to moderate (12-16), moderate (8-11) and severe (5-7).? For the purpose of this study, ED was defined as an IIEF-5 score $<22$.

Patients were also screened for depression. This was performed using the Beck Depression Inventory (BDI). ${ }^{8}$ This instrument assesses the presence of depressive symptoms experienced by the patient within the past week. It is composed of 21 questions. Each question is scored on a 4-point scale, ranging from no impairment (0) to severe impairment (3). Mild depressive symptoms are indicated with a score $>10$ and severe depressive symptoms are indicated with a score $>30 .{ }^{8}$ This tool has been validated and commonly used in patients with end stage renal disease. ${ }^{9}$ For the purpose of this study, depression was defined as a BDI score of $>10$.

We performed a retrospective chart review for all patients enrolled in this study. Patients' characteristics, including age, presence of a partner, time on dialysis, method of dialysis, duration of current transplant, number of transplants, vessel used for renal artery anastomosis, smoking, diabetes mellitus, hypertension, dyslipidemia, immunosuppressive regimen, rejection episodes, antihypertensive medications and most recent serum creatinine level, were obtained. Serum testosterone was also determined for each patient to determine whether any of the patients were hypogonadal.

We compared characteristics of patients who had ED with characteristics of those who did not have ED with respect to depression, testosterone and the above-mentioned variables obtained from the chart review. Statistical relations between erectile status and patient characteristics were identified with Student's $t$ tests for quantitative variables and chi-squared tests for qualitative variables. A 2-tailed $p$ value $<0.05$ was considered statistically significant. Only those statistically significant factors from the univariate analysis were studied further in a multivariate analysis. We performed multivariate analysis between the potential prognostic factors and the presence of ED using logistic regression. Variables associated with the outcome at $p<0.05$ were included in the final model. The statistical analyses were performed using SPSS 14.0 for Windows (SPSS Inc., Chicago, III.). We obtained informed consent from all patients, and the study was approved by the Institutional Research Ethics Board.

\section{Results}

This study involved 55 patients. Table 1 and Table 2 describe the clinical characteristics of these patients. The average age of the patients was 50 years old (range 19.5-75.6 yr) and almost all of the patients had a partner $(n=52)$. The causes of renal failure are listed in Table 3. Most (67\%) of these patients required pre-transplant dialysis, with a mean duration of dialysis of less than 1 year (9.8 mo). The mean duration of the current transplant was 7.9 years, with $84 \%$ of patients only requiring a single transplant.

\begin{tabular}{lccc}
\hline \multicolumn{3}{l}{ Table 1: Clinical characteristics of the study population } \\
\hline \multicolumn{4}{c}{ Group; mean (and SD) } \\
\cline { 2 - 4 } Characteristic & Total, $n=55$ & ED, $n=28$ & No ED, $n=27$ \\
\hline Duration of transplant, yr & $7.9(7.6)$ & $7.0(7.6)$ & $8.8(7.5)$ \\
Time on dialysis, mo & $9.8(13.9)$ & $9.9(12.6)$ & $9.6(15.3)$ \\
Creatinine, $\mu \mathrm{mol} / \mathrm{L}$ & $177.4(89.7)$ & $159.5(59.0)$ & $193.6(111.8)$ \\
Testosterone, $\mathrm{nmol} / \mathrm{L}$ & $15.7(5.2)$ & $16.4(4.7)$ & $15.2(5.8)$ \\
No. of hypertension medications & $2.2(1.0)$ & $2.1(1.1)$ & $2.2(0.9)$ \\
\hline $\mathrm{SD}=$ standard deviation; $\mathrm{ED}=$ erectile dysfunction. & & \\
\hline
\end{tabular}


Almost all (96\%) of the patients had hypertension and the mean number of antihypertensive medications required was 2.2. The most commonly used antihypertensive medication was a calcium channel blocker $(n=36)$, with an angiotensinconverting enzyme inhibitor (ACEI) or angiotensin II receptor blocker (ARB) $(n=35)$ and $\beta$-blocker $(n=32)$ being commonly used as well. Cyclosporine based immunosuppression was employed in 29 patients. No patients had low serum testosterone (normal $=8.4-28.7 \mathrm{nmol} / \mathrm{L}$ ) and the majority $(89 \%)$ of patients had an elevated creatinine (> $110 \mu \mathrm{mol} / \mathrm{L})$, although all patients had a stable creatinine.

ED was identified in 28 of the patients, representing $51 \%$ of the study population. Of these patients, $11(20 \%)$ had mild ED, 6 patients $(11 \%)$ had mild-to-moderate ED, 4 patients (7\%) had moderate ED and 7 patients (13\%) had severe ED. Patients with ED were offered treatment in the form of a Phosphodiesterase-5 (PDE-5) inhibitor. More patients with ED were older than 50 years of age, compared with those who did not have ED (64\% v. $26 \%, p=0.004)$. There was a higher prevalence of diabetes mellitus (39\% v. $11 \%, p=0.02)$ in patients with ED compared with those patients without ED. In addition, more patients with ED were depressed, compared with those patients who did not have ED ( $29 \%$ v. $7 \%, p=0.04)$. These 3 factors were significantly associated with ED on univariate analysis and this relation was confirmed on multivariate analysis (Table 4). There were no other statistically significant differences between patients with ED and those without ED. The relation between the presence of these 3 risk factors and ED are illustrated graphically in Figure 1. The proportion of patients with ED ranged from $21 \%$ to $100 \%$ depending on the number of risk factors present. The presence of more risk factors was associated with a higher risk of ED.

\section{Discussion}

The effect of renal transplantation on patients with $\mathrm{ED}$ is controversial. Earlier studies have determined the prevalence of ED following renal transplant to be between $54 \%$ and $66 \% .^{10}$ The prevalence of ED in our study, with a sample size of 55 patients, was $51 \%$, which is consistent with that reported in the current literature. Overall, $11(20 \%), 6$ $(11 \%), 4(7 \%)$ and $7(13 \%)$ patients had mild, mild-moderate, moderate, and severe ED, respectively.

Increased age is a well-known risk factor for $E D$. The importance of increased age on the prevalence of ED in the renal transplant population has been verified in several studies., ${ }^{5,10,11}$ In Malavaud and colleagues' study of 271 post renal transplant patients, the average age of patients with ED was 49.2 years old, compared with 44.5 years old for patients without ED. ${ }^{5}$ The mean age of patients with ED after renal transplant in another study was 43.6 years old, compared with 34.9 years old in patients without ED. ${ }^{10}$ They found that patients who were over 40 years of age were 3.1 times more likely to have $\mathrm{ED} .{ }^{10}$ In the current study, the mean age of patients with ED was 55.0 years and the

Table 2: Clinical characteristics of the study population

\begin{tabular}{|c|c|c|c|}
\hline \multirow[b]{2}{*}{ Characteristics } & \multicolumn{3}{|c|}{ Group; no. (and \%) of subjects } \\
\hline & Total, $n=55$ & $\mathrm{ED}, n=28$ & No ED, $n=27$ \\
\hline Age $\geq 50 \mathrm{yr}^{*}$ & $25(45)$ & $18(64)$ & $7(26)$ \\
\hline Partner & $52(95)$ & $26(93)$ & $26(96)$ \\
\hline Living donor & $25(45)$ & $10(37)$ & $15(56)$ \\
\hline Living related donor & $20(36)$ & $9(32)$ & $11(41)$ \\
\hline Internal iliac & $7(13)$ & $3(11)$ & $4(15)$ \\
\hline First transplant & $46(84)$ & $26(93)$ & $20(74)$ \\
\hline Dialysis & $37(67)$ & $16(57)$ & $21(78)$ \\
\hline Hemodialysis & $19(35)$ & $8(29)$ & $11(41)$ \\
\hline Hypertension & $53(96)$ & $27(96)$ & $26(96)$ \\
\hline Diabetes mellitust & $14(25)$ & $11(39)$ & $3(11)$ \\
\hline Dyslipidemia & $43(78)$ & $23(82)$ & $20(74)$ \\
\hline Smoker & $11(20)$ & $5(18)$ & $6(22)$ \\
\hline Organ rejection & $16(29)$ & $7(25)$ & $9(33)$ \\
\hline \multicolumn{4}{|l|}{ Medication } \\
\hline$\beta$-blocker & $32(58)$ & $15(54)$ & $17(63)$ \\
\hline ACEI or ARB & $35(64)$ & $18(64)$ & $17(63)$ \\
\hline CCB & $36(65)$ & $16(57)$ & $20(74)$ \\
\hline HCTZ & $10(18)$ & $6(21)$ & $4(15)$ \\
\hline$\alpha$-blocker & $6(11)$ & $3(11)$ & $3(11)$ \\
\hline Cyclosporine & $29(53)$ & $17(61)$ & $12(44)$ \\
\hline Tacrolimus & $22(40)$ & $9(32)$ & $13(48)$ \\
\hline Mycophenolate & $29(53)$ & $17(61)$ & $12(44)$ \\
\hline Sirolimus & 5 (9) & $2(7)$ & $3(11)$ \\
\hline Prednisone & $40(73)$ & $20(71)$ & $20(74)$ \\
\hline Azathioprine & $6(11)$ & $2(7)$ & $4(15)$ \\
\hline Depression $¥$ & $10(18)$ & $8(29)$ & $2(7)$ \\
\hline
\end{tabular}

$\mathrm{ED}=$ erectile dysfunction; $\mathrm{ACEI}=$ angiotensin-converting enzyme inhibitor; $\mathrm{ARB}=$ angiotensin II receptor blocker; $\mathrm{CCB}=$ calcium channel blocker; $\mathrm{HCTZ}$ = hydrochlorothiazide.

${ }^{*} p=0.004\left(\chi^{2}\right.$ test)

$\dagger p=0.02\left(\chi^{2}\right.$ test $)$

$\mp p=0.04$ ( $\chi^{2}$ test $)$ 
mean age of patients who did not have ED was 45.2 years. The median age of patients with ED was 53 years and the median age of patients who did not have ED was 46 years. Patients who were over age 50 years were 7.2 times more likely to have ED than their younger counterparts.

Diabetes is a risk factor commonly associated with ED. ${ }^{12}$ The odds ratio of having ED if a patient has diabetes is 1.9 to 4 times greater than those patients without diabetes. ${ }^{12}$ The cause of diabetic ED is multifactorial and would include an acceleration of vascular disease and a neuropathic mechanism. ${ }^{13}$ The impact of diabetes on erectile function in the renal transplant population was documented by El-Bahnasawy and colleagues. ${ }^{10}$ They noted a relative risk of 3 for ED in patients with diabetes, compared with patients who did not have diabetes. In our study, the presence of diabetes was a significant risk factor for developing $E D$. The odds ratio for ED in patients with diabetes was 6.1, compared with patients without diabetes.

The association between depression and ED is well known. ${ }^{14}$ The occurrence of one disorder may

\begin{tabular}{|c|c|c|}
\hline Cause of renal failure & $\begin{array}{c}\text { No. of } \\
\text { patients } \\
\text { with ED }\end{array}$ & $\begin{array}{c}\text { No. of } \\
\text { patients } \\
\text { without ED }\end{array}$ \\
\hline Glomerulonephritis & 4 & 10 \\
\hline Diabetes mellitus & 9 & 3 \\
\hline IgA nephropathy & 3 & 6 \\
\hline Polycystic kidney disease & 4 & 3 \\
\hline Hypertension & 3 & 0 \\
\hline Interstitial nephritis & 1 & 0 \\
\hline Wegener's granulomatosis & 1 & 0 \\
\hline Posterior urethral valves & 0 & 1 \\
\hline Congenital hydronephrosis & 0 & 1 \\
\hline Alport's syndrome & 1 & 0 \\
\hline Hemolytic uremic syndrome & 1 & 0 \\
\hline Sarcoidosis & 1 & 0 \\
\hline Unknown & 0 & 3 \\
\hline
\end{tabular}

Table 4: Risk factors for erectile dysfuction

\begin{tabular}{lccc}
\hline Variable & OR & $95 \% \mathrm{Cl}$ & $p$ value \\
\hline Age $\geq 50$ & 7.221 & $1.837-28.384$ & 0.005 \\
Diabetes mellitus & 6.127 & $1.185-31.682$ & 0.031 \\
Depression & 9.444 & $1.372-65.020$ & 0.023 \\
\hline OR = odds ratio; $\mathrm{Cl}=$ confidence interval. & & \\
\hline
\end{tabular}

cause, result from or exacerbate the other. ${ }^{14}$ In a study assessing the incidence of depressive symptoms in patients with ED, men with ED were 2.6 times more likely to report depressive symptoms than those without ED. ${ }^{13}$ In addition, patients who described depressive symptoms were less likely to be compliant with treatment for $\mathrm{ED}$, resulting in a poorer outcome. ${ }^{13}$ In a quality of life study including 50 renal transplant recipients, the mean BDI score was 8.1 (standard deviation 7.8).$^{15}$ Overall, $20 \%$ of the patients had a BDI score $>12 .{ }^{15}$ In the current study, there was a higher prevalence of depression in patients with ED. In the entire cohort, 10 patients $(18 \%)$ were identified by the BDI as having at least mild depression. Overall, 8 of these patients had ED and 2 did not have ED. Therefore, $29 \%$ of patients who had ED also had concomitant depression. The significance of this finding was confirmed on multivariate analysis. To the best of our knowledge, this is one of the first studies to assess the risk of ED and concomitant depression in men who have received a renal transplant. It is unknown whether the ED caused or worsened the patients' depression or if the depression caused or worsened the patients' erectile dysfunction. It is also unknown whether improvement in the patients' erectile function would ultimately improve their depression or vice versa.

Other studies have identified time on dialysis as a risk factor for ED in the renal transplant population. ${ }^{5,11}$ This was not confirmed in our study. Other studies have also not documented the relation between duration of dialysis and ED. ${ }^{10}$ However, it should be noted that the patients in our current study only required dialysis for a short duration (mean $9.8 \mathrm{mo}$ ), compared with studies that identified a relation between dialysis status and erec-

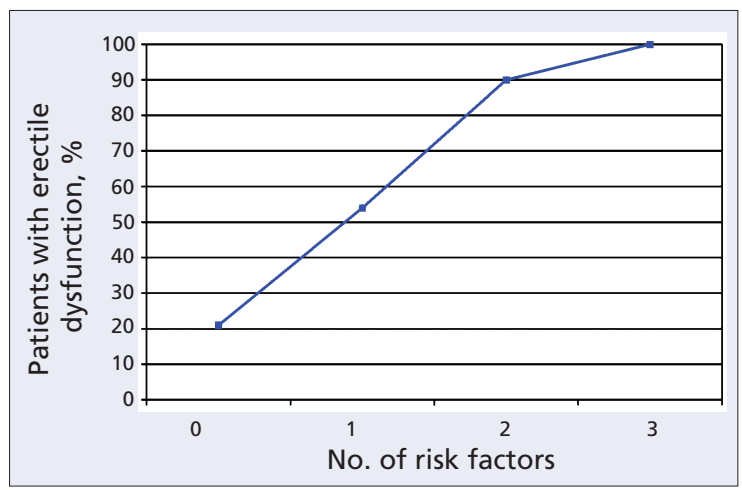

Fig. 1. Number of risk factors and presence of erectile dysfunction. 
tile function (28.5 mo to $7.9 \mathrm{yr})^{5,11}$ This short duration of dialysis is a function of the high proportion of pre-emptive living donor transplants and the availability of kidneys at our institution, which is a major tertiary care centre for the Atlantic provinces.

Chronic renal failure can produce testicular damage, impair spermatogenesis and decrease testosterone production ${ }^{2}$; thus, renal transplant recipients could be at risk for hypogonadism, which is a potentially treatable cause of ED. It is interesting to note that none of the patients in this study were hypogonadal. This is possibly owing to the normalization of the hypothalamic-pituitary-gonadal axis that occurs with renal transplantation.'

Limitations to this study include its crosssectional design. A longitudinal study with preand post-transplant data would be ideal to help elucidate the role of renal transplantation for ED in patients with renal failure. Follow-up would also be useful to determine the success rates of the prescribed treatment for ED. In addition, it would be useful to characterize the prevalence of depression in these patients before and after transplant and its relation to erectile function. However, we do believe that important information can be learned from this cross-sectional study. Further, within this cross-sectional study we felt that it would be inappropriate to question the patients concerning their preoperative erectile function status or depression as there would be significant recall bias. Another limitation to the study is its small sample size. This could have the effect of not identifying a potential prognostic factor for patients with ED following renal transplant. This study is, however, one of the larger studies to explore ED in the renal transplant population.

\section{Conclusion}

ED remains a common problem in the renal transplant population. The cause of ED is multifactorial, with increasing age and the presence of diabetes mellitus and depression increasing the risk of ED. As clinicians, we must be aware of the high prevalence of $\mathrm{ED}$, particularly in patients with these risk factors, to ensure that proper attention is given to this major quality of life issue. We suggest during the routine follow-up of renal transplant recip- ients, particularly those who are older and those who have diabetes mellitus, or both, that they be questioned about the presence of ED. In addition, in patients with $\mathrm{ED}$, coexisting depressive symptoms should be elicited as they may have implications on the success rate of current ED therapies and quality of life outcomes.

From the Departments of *Urology and †Medicine, Dalhousie University and Queen Elizabeth II Health Sciences Centre, Halifax, NS

This article has been peer reviewed.

Competing interests: Dr. Waleed Alkhudair received an unrestricted grant from Pfizer Canada in the amount of $\$ 30000$.

\section{References}

1. Handelsman DJ, Dong Q. Hypothalamo-pituitary gonadal axis in chronic renal failure. Endocrinol Metab Clin North Am 1993;22:145-61.

2. Burgos FJ, Pascual J, Gomez V, et al. Effect of kidney transplantation and cyclosporine treatment on male sexual performance and hormonal profile: a prospective study. Transplant Proc 1997;29:227-8.

3. Stewart RS. Psychiatric issues in renal dialysis and transplantation. Hosp Community Psychiatry 1983;34:623-8.

4. Morgentaler A. Male impotence. Lancet 1999;354:1713-8.

5. Malavaud B, Rostaing L, Rischmann P, et al. High prevalence of erectile dysfunction after renal transplantation. Transplantation 2000;69:2121-4.

6. El-Bahnasawy MS, El-Assmy A, Dawood A, et al. Effect of the use of internal iliac artery for renal transplantation of penile vascularity and erectile function: a prospective study. J Urol 2004; 172:2335-9.

7. Rosen RC, Cappelleri JC, Smith MD, et al. Development and evaluation of an abridged, 5-titem version of the International Index of Erectile Function (IIEF-5) as a diagnostic tool for erectile dysfunction. Int I Impot Res 1999;11:319-26.

8. Beck AT, Beck RW. Screening depressed patients in family practice: a rapid technique. Postgrad Med 1972;52:81-5.

9. Craven JL, Rodin GM, Littlefield C. The Beck Depression Inventory as a screening device for major depression in renal dialysis patients. Int J Psychiatry Med 1988;18:365-74.

10. El-Bahnasawy MS, E-Assmy A, E-Sawy E, et al. Critical evaluation of the factors influencing erectile function after renal transplantation. Int I Impot Res 2004; 16:521-6.

11. Rebollo P, Ortega F, Valdes C, et al. Factors associated with erectile dysfunction in male kidney transplant recipients. Int J Impot Res 2003;15:433-8.

12. Costabile RA. Optimizing treatment for diabetes mellitus induced erectile dysfunction. J Urol 2003;170:S35-9.

13. Shabsigh R, Klein LT, Seidman $S$, et al. Increased incidence of depressive symptoms in men with erectile dysfunction. Urology 1998;52:848-52.

14. Nurnberg HG, Seidman SN, Gelenberg AJ, et al. Depression, antidepressant therapies, and erectile dysfunction: clinical trials of sildenafil citrate (Viagra) in treated and untreated patients with depression. Urology 2002;60:58-66.

15. Shah VS, Ananth A, Sohal GK, et al. Quality of life and psychosocial factors in renal transplant recipients. Transplant Proc 2006;38:1283-5.

Correspondence: Dr. Waleed K. Alkhudair, Queen Elizabeth II Health Sciences Centre, Rm. 204, 5-South, Victoria Building, VG Site, 1278 Tower Rd., Halifax NS B3H 2Y9; Waleed.Alkhudai@dal.ca 